\title{
L'ACTION PUBLIQUE Contaminants chimiques des aliments : évaluation et gestion des risques
}

\section{Public intervention Chemical contaminants in food: evaluation and risk management}

Oléagineux, Corps Gras, Lipides. Volume 7, Numéro 5, 412-5, Septembre - Octobre 2000, Dossier : Sécurité sanitaire des aliments et industrie

Auteur(s) : Jean-François NARBONNE, UMR CNRS 5472, Université Bordeaux-1, avenue des Facultés, bâtiment Biologie animale, 33405 Talence Cedex.

Résumé : La sécurité des aliments s'appuie sur l'évaluation des risques, la traçabilité (de la fourche à la fourchette) et la veille sanitaire. Suite aux crises récentes, ce domaine connaît aujourd'hui une profonde transformation des liens entre citoyens, opérateurs privés, pouvoirs publics, médias, éducateurs, corps médical et recherche, le consommateur étant désormais placé au centre du système.

Mots-clés : évaluation des risques.

Summary : Food safety is based on the assessment of risks, the traceability (from the farm to fork) and monitoring. These sectors are knowing a deep evolution, with new relashionship between citizen, food industry, politics, medias, educational system, medical professions, and research, the consumer being now positioning at the center of the system.

Keywords : risks assessment, traceability, monitoring.

\section{ARTICLE}

Les relations entre l'aliment et la santé du consommateur ont fortement évolué au cours des cinquante dernières années. Au sortir de la Seconde Guerre mondiale le terme de sécurité alimentaire concernait la disponibilité de la nourriture pour prévenir les famines. Il s'agissait alors de gérer au niveau mondial les stocks d'aliments, rôle qui a été dévolu à la FOA/OMS. Les changements spectaculaires dans les pratiques en agriculture et élevage ayant assuré la disponibilité des aliments (au moins dans les pays riches), les développements ont porté sur des aspects nutrition/sécurité. On a ainsi assisté au développement d'aliments adaptés à des situations physiologiques particulières : aliments pour nourrissons et enfants, aliments allégés, aliments fonctionnels retardant la sénescence physiologique ou pathologique. En outre, on a cherché à réduire les maladies provoquées par l'alimentation, en particulier celles dues aux altérations microbiologiques. La sécurité a concerné essentiellement I'hygiène des aliments avec l'établissement de procédures visant à éliminer ou à réduire les flores pathogènes. Des techniques de conservation permettant le maintien d'une bonne qualité microbiologique pendant des temps de plus en plus longs ont été développées. Ces nouvelles exigences ont entraîné des changements profonds dans les filières alimentaires, particulièrement au niveau de la transformation et de la distribution (marche en avant, usines " ultrapropres ", chaîne du 
froid...). Ces mesures ont eu comme résultat une diminution spectaculaire des cas d'intoxications alimentaires. Plus récemment, l'intérêt des consommateurs s'est porté sur les risques chimiques, plus complexes à cerner ; les effets potentiels étant le plus souvent à long terme, il est très difficile d'établir des liens de causalité (maladies apparaissant 20 ou 40 ans après exposition, troubles affectant la descendance). Les événements récents qui ont touché à la sécurité alimentaire (dioxine, nitrates, métaux lourds, nitrosamines, Coca Cola, Erika...) ont eu un retentissement important dans les médias et ont particulièrement sensibilisé le consommateur et l'ensemble de la filière agroalimentaire. Ainsi, contrairement aux risques microbiologiques, la gestion des risques chimiques doit être précédée d'une phase d'évaluation mobilisant des experts de disciplines diverses. L'importance de cette évaluation a eu comme conséquence une restructuration des instances qui, jusqu'à présent, étaient dispersées au sein de plusieurs ministères. La création de l'Agence française de sécurité sanitaire des aliments a été suscitée par les nouvelles exigences d'une évaluation de l'ensemble des risques sanitaires liés à l'alimentation.

\section{Évaluation des risques}

La définition de la toxicité a évolué au cours de l'histoire de la chimie et de la biologie. Un toxique fut d'abord un poison entraînant rapidement la mort. L'exemple le plus célèbre étant celui de Socrate buvant la ciguë. Le concept de toxicité a ensuite évolué vers la notion d'effets sublétaux entraînant des maladies puis, de façon plus subtile, induisant des effets néfastes sur la santé. Avec le développement de la biologie cellulaire et moléculaire, on considère à l'heure actuelle comme effet toxique une action perturbatrice sur la régulation des mécanismes cellulaires ou même une fixation sur un récepteur. On peut dire que la toxicité d'un produit traduit la nature des dommages (par exemple induction de tumeurs cancéreuses ou neurotoxicité) susceptibles d'affecter un individu exposé. Elle entre donc dans la notion de danger.

Parallèlement, l'évolution des techniques analytiques a aussi entraîné une diminution considérable des seuils de détections qui se situent à l'heure actuelle, pour certains contaminants, alimentaires à un niveau de l'ordre du femto- gramme ${ }^{1}$. Ceci pose le problème de la différenciation entre le danger et le risque. Dans le passé, les seuils de détection étant très élevés, le fait de montrer la présence d'un contaminant dans un aliment signifiait en même temps que sa concentration était forte, donc que l'exposition et le risque étaient élevés. Aujourd'hui, la détection de traces d'un contaminant dans un aliment peut indiquer, au contraire, une faible exposition et donc n'implique pas obligatoirement un risque pour le consommateur. Le danger est représenté par le vecteur (par exemple le composé chimique) et les dommages potentiels sur la santé. Il est ensuite nécessaire de caractériser ce danger en fixant la dose et la durée d'exposition à partir desquelles l'effet toxique apparaît. Ce seuil est en général défini par la dose journalière tolérable (DJT) calculée par application de facteurs de sécurité à partir des doses sans effets observées dans les expérimentations animales. Les données cliniques et épidémiologiques viennent, quand elles sont disponibles, étayer les calculs de la DJT. Dans le cas de cancérogènes, les experts américains utilisent des modèles d'évaluation quantitative des risques et calculent une dose virtuellement sûre (DVS) exprimée par la quantité pouvant induire au plus un cancer supplémentaire pour 1000000 d'individus pour la vie entière. 
Le risque est la probabilité d'être dans une situation où des dommages pour la santé peuvent apparaître. Pour évaluer le risque lié à un contaminant, il faut donc estimer l'exposition de l'ensemble de la population ou de fractions de population. Pourront être considérés comme en situation de risque potentiel, d'une part, les consommateurs exposés à des doses supérieures à la DJT pendant de longues périodes et, d'autre part, des individus pouvant présenter une sensibilité particulière en raison de leur état physiologique ou pathologique. Une bonne évaluation des risques est donc fondée non seulement sur un solide dossier toxicologique, mais aussi sur de nombreux éléments de connaissance tels que la distribution des teneurs en contaminant dans les aliments, les modèles de consommation (population générale, consommateurs réguliers ou extrêmes, enfants...), l'état sanitaire des individus. II faut aussi signaler les difficultés d'évaluation du risque dans le cas de dépassement temporaire de la DJT. Quelques exemples récents illustrent ces difficultés.

\section{Exemples d'évaluation des risques alimentaires}

* Dans le cas de la contamination des mollusques sur les côtes bretonnes par le fioul lourd déversé à la suite du naufrage de l'Erika, deux opérations parallèles ont été effectuées :

- une évaluation de la contamination par les hydrocarbures aromatiques polycycliques (HAP) des mollusques issus des zones contaminées, comparativement aux teneurs mesurées dans des échantillons prélevés dans les années antérieures à la pollution et considérées comme " bruit de fond " ;

- une estimation des risques correspondant à différents scénarios de consommation de produits de la mer.

Dans un premier temps, l'identification du danger a permis de sélectionner les hydrocarbures aromatiques polycycliques présentant un potentiel cancérigène reconnu (18 composés de la liste EPA dont 6 majeurs de la liste OMS). Pour estimer ensuite les niveaux possibles d'exposition, les données historiques de contamination des poissons et mollusques (sources Ifremer et programme européen Biomar LPTC) ont été comparées à celles issues de dosages d'organismes prélevés dans les zones contaminées et ayant été au contact du fioul lourd. De plus, les données sur la consommation des animaux marins en question ont été réunies.

Dans un deuxième temps, une étude a été effectuée pour évaluer le niveau de risque potentiel de cancérogenèse en fonction, d'une part, de modèles d'évaluation quantitative de risques utilisés par certains pays européens et, d'autre part, des données moyennes de consommation journalière des produits de la mer par le consommateur français. Ainsi, la consommation de produits non contaminés (teneurs dites naturelles ou bruit de fond) fait apparaître un risque significatif pour une ingestion de 500 huîtres par jour pendant la vie entière. Pour les mollusques les plus contaminés, un risque significatif correspond à la consommation de 5 huîtres par jour pendant la vie entière. Ces calculs ont donc permis d'établir les valeurs limites comme options de gestion de risque.

* La crise dite " de la dioxine " en Belgique, qui a mobilisé l'attention des consommateurs en juin 1999, est en fait la conséquence de dysfonctionnements dans l'évaluation et la gestion des risques sanitaires alimentaires dans ce pays. En effet, l'erreur de diagnostic sur la nature de la contamination a entraîné un retard de deux mois dans l'identification de la source (contamination par des PCB d'huiles industrielles). Ainsi, du fait du délai nécessaire pour l'analyse par spectrométrie de masse 
haute résolution ( 3 semaines pour la première série d'analyses, puis autant pour la confirmation), les produits contaminés avaient été consommés. Si les PCB avaient été recherchés (temps d'analyse de deux jours par chromatographie en phase gazeuse et détection par capture d'électrons), la contamination aurait été détectée avant la distribution des produits. D'ailleurs, en conséquences de la crise de juin 1999, un plan de surveillance des PCB dans l'alimentation animale a mis en évidence deux épisodes de contamination, mais avec intervention précoce au niveau des élevages. II faut toujours se rappeler que le risque est lié à l'exposition et que l'exposition est liée au taux de contamination de l'aliment et à son niveau de consommation.

Dans le cas de la dioxine, deux types de démarches ont été effectués pour situer les niveaux de risque acceptables.

Une première démarche (choisie par les experts français), qui est fondée sur la valeur haute de la DJT et proposée par l'OMS, peut être appelée " classique ". En effet, les experts français considèrent que la valeur de $1 \mathrm{pg} / \mathrm{kg} / \mathrm{j}$ est la dose moyenne ne devant pas être dépassée sur l'ensemble de la vie et que $4 \mathrm{pg} / \mathrm{kg} / \mathrm{j}^{2}$ est une valeur limite pour des temps plus courts. C'est donc cette valeur haute de la DJT qui a servi de base à une proposition de valeur limite d'exclusion pour chaque aliment en fonction de son niveau de consommation.

Les experts hollandais proposent une approche différente qui prend comme base de l'évaluation du risque la charge corporelle en dioxines. En effet, ces composés liposolubles et très peu dégradés s'accumulent dans les graisses corporelles et la concentration augmente avec l'âge. C'est donc moins la valeur de l'apport journalier que la courbe d'accumulation qui est considérée comme base de calcul du risque. À partir de ce type de raisonnement, pour un niveau en dioxines de $60 \mathrm{pg}$ ITEO/g de matière grasse, le temps nécessaire pour doubler la charge corporelle d'un individu est de 30000 jours de consommation lorsqu'il s'agit d'œufs contaminés et de seulement 270 jours si la contamination concerne la viande et les produits laitiers. Cette démarche est intéressante car elle donne une estimation du temps maximum disponible pour résoudre une crise.

L'ensemble de ces exemples montre que les profondes évolutions dans la détection des dangers et dans l'évaluation des risques donnent de nouveaux outils pour la gestion des risques, mais aussi pour la communication avec les consommateurs et l'ensemble des opérateurs des filières alimentaires.

\section{Évaluation des risques : un service public}

L'identification des dangers et l'évaluation de l'exposition des consommateurs à ces dangers ont toujours été considérées en France comme les plus solides fondements des décisions des pouvoirs publics dans le domaine de la gestion du risque alimentaire. Une telle évaluation est, par définition, pluridisciplinaire et fait appel à de nombreuses compétences et structures dans le domaine de la santé, de la microbiologie, de la toxicologie, de la nutrition, de l'épidémiologie, de la chimie analytique... II a donc fallu organiser le rôle des différents acteurs de l'expertise. En France, le Conseil supérieur d'hygiène publique placé sous l'égide du ministère de la Santé avait en charge, par l'intermédiaire de sa section alimentation et nutrition, d'effectuer l'évaluation des risques liés à l'alimentation et de proposer aux pouvoirs publics des options de gestion. Mais d'autres organismes intervenaient aussi comme l'Académie de médecine, les groupes d'experts du ministère de l'Agriculture et de la répression des fraudes. Il est évident que cette étape essentielle ne doit pas rester obscure pour les autres partenaires (gestionnaires publics, industriels et consommateurs) car 
c'est la connaissance des risques qui permet une bonne gestion et une information claire. II s'agit aussi, pour le consommateur, d'être informé objectivement car c'est lui qui, en définitive, accorde sa confiance à un système par son acte d'achat qui constitue en quelque sorte un " contrat social ". Aussi le parlement a-t-il créé, par la loi du $1^{\text {er }}$ juillet 1998, l'Agence française de sécurité sanitaire des aliments (AFSSA) qui constitue dorénavant l'outil national d'évaluation des risques sanitaires et nutritionnels des aliments. Le dispositif de veille sanitaire mis en place par cette loi comprend en outre I'Institut de veille sanitaire (IVS) qui remplace I'ancien Réseau national de santé publique (RNSP) et prévoit la coordination de l'AFSSA, de l'IVS et de l'Agence française de sécurité sanitaire des produits de santé (sang, médicaments, etc.) par le Comité national de sécurité sanitaire, présidé par le ministre de la Santé. L'ensemble du dispositif d'évaluation doit être rapide et efficace en cas de crise car la mise en application du système de précaution implique une large mise sous séquestre des produits ou des établissements suspects, mais qui ne se conçoit que si une évaluation rapide du risque permet de libérer les produits ou les établissements ne présentant pas de risques significatifs pour les consommateurs. Dès sa mise en place, I'AFSSA a été confrontée à une succession de crises qui ont montré son efficacité.

En dehors des périodes de crise, I'AFSSA procède à des analyses de fond sur des problèmes émergeants et peut bénéficier d'expertises collectives de l'INSERM pour étayer les connaissances sur les dangers. L'estimation du risque est réalisée par des évaluations d'exposition fondées sur des études du type " panier de la ménagère " ou du type "repas totaux dupliqués ". Les données de consommation sont collectées par l'Observatoire de consommations alimentaires (OCA) qui a été rattaché à I'AFSSA. Les données analytiques sont apportées par les services de contrôle (services vétérinaires, DGCCRF, DRASS) ou même par les organismes professionnels à l'occasion de plans de surveillance et, dans certaines conditions, de plans de contrôle.

\section{Gestion des risques}

Cette évaluation va servir de base aux options de gestion des risques, qui a pour but d'assurer la sécurité des consommateurs. En fait, la gestion du risque est l'application de mesures visant à supprimer ou à limiter l'exposition afin d'éliminer ou réduire les risques à des niveaux acceptables par la société, pour l'ensemble ou une partie de la population. La notion d'acceptabilité du risque par la société fait évidemment intervenir des éléments de la balance bénéfices/risques. Dans le cas où les éléments scientifiques sont insuffisants pour procéder à une évaluation argumentée du risque, I'application du principe de précaution implique l'abstention qui peut se traduire par exemple par un moratoire, c'est-à-dire un délai nécessaire à l'acquisition des données minimales pour permettre l'évaluation du risque. Ici la balance bénéfice/risque vient moduler le principe de précaution, la société étant prête à accepter un risque potentiel pour un bénéfice d'usage évident (par exemple, les téléphones portables). Au contraire, si les bénéfices d'une technologie n'apparaissent pas évidents pour le consommateur, le principe de précaution sera alors privilégié (exemple des plantes OGM). En fait, la gestion des risques doit garantir l'innocuité des produits mis sur le marché, mais la responsabilité est à la fois individuelle et collective. Une partie de la gestion des risques incombe à la collectivité (locale, nationale ou internationale) qui définit par l'arsenal législatif les " règles du jeu ", de façon à éviter les atteintes à la santé publique. Cet arsenal peut faire appel à toute une gamme de mesures incluant par exemple des valeurs limites, guides ou cibles. 
L'évaluation des risques est effectuée par des instances nationales et internationales et la gestion des risques est effectuée par les pouvoirs publics chargés d'édicter la loi et de contrôler son application.

\section{Services publics et gestion des risques}

La gestion du risque alimentaire est une mission de service public. La responsabilité en est confiée au pouvoir exécutif qui fait ses choix à la lumière des expertises effectuées, afin d'assurer la maîtrise du risque alimentaire. II appartient à l'administration de définir " les règles du jeu " et de vérifier qu'elles sont bien respectées. La gestion du risque alimentaire est donc une prérogative politique qui consiste à mettre en adéquation les recommandations formulées par les experts chargés de l'évaluation scientifique des risques, les moyens de toute nature que la collectivité, les entreprises et les producteurs peuvent consacrer à la maîtrise de ces risques, les objectifs en termes de niveau de sécurité défini par le gouvernement et à procéder à une large information du public. Le processus de décision doit donc désormais s'appuyer sur des consultations ouvertes. La Direction générale de l'alimentation exerce désormais exclusivement cette mission.

Le contrôle du respect des réglementations relatives à l'hygiène des aliments est ensuite mis en œuvre par les services déconcentrés de l'État : les services vétérinaires départementaux, les services régionaux de la protection des végétaux, les DDCCRF et les DDASS pour certains aspects (comme l'eau et les infections humaines). À l'initiative des préfets de département, les pôles de compétence regroupant tous les services de l'État intervenant en matière de sécurité de la filière agricole et alimentaire sont désormais nombreux. Les contrôles sur le terrain contribuent à assurer la sécurité des produits. En effet, les agents de contrôles, et notamment ceux des services vétérinaires, sont chargés de vérifier l'application par les professionnels des réglementations en matière d'hygiène des aliments. Ce contrôle se situe à deux niveaux différents. Le premier, traditionnel, consiste en la vérification du respect des prescriptions classiques, la conception, l'aménagement, l'équipement et l'entretien des locaux, le comportement du personnel et sa propreté. Le second niveau découle des principes des auto-contrôles et s'exerce en vérifiant ceux-ci.

Les services chargés du contrôle de terrain sont épaulés par des laboratoires publics nationaux ou locaux. Des laboratoires interprofessionnels et des laboratoires privés agréés par le MAP peuvent également être sollicités pour des analyses de routine.

Certaines enquêtes touchant plusieurs départements nécessitent une organisation et une harmonisation dépassant le cadre du seul département. Pour ce faire, le ministère de l'Agriculture a créé la Brigade nationale d'enquêtes vétérinaires et sanitaires dont le champ de compétences géographiques couvre la totalité du territoire français. Au total, plus de 6000 personnes participent en France à la veille sanitaire en matière d'alimentation, dont environ 4000 au sein du ministère de l'Agriculture et de la Pêche.

\section{Gestion des risques par les opérateurs}

La gestion pratique relève toutefois de la responsabilité individuelle des opérateurs qui doivent donc, d'une part, respecter les règles du jeu collectives et, d'autre part, mettre tout en œuvre pour assurer l'innocuité des produits mis sur le marché. En cas de crise, l'opérateur sera donc jugé en fonction des méthodes et des moyens mis en œuvre. La responsabilité des professionnels est donc engagée quant à leurs pratiques et à leurs conséquences, ce qui est un des éléments fondamentaux de la sécurité 
alimentaire. II y a donc une obligation pour les entreprises agro-alimentaires qui se traduit par la mise en place d'auto-contrôles fondée sur les principes de la méthode HACCP, introduite par la loi du 19 mai 1998. L'absence ou l'inefficacité des auto-contrôles peut entraîner des sanctions administratives importantes allant de la saisie des denrées produites jusqu'à la fermeture de l'établissement. Pour se prévenir des conséquences judiciaire, économique et médiatique d'une crise, l'opérateur devra donc pouvoir démontrer qu'une procédure de maîtrise des risques a été mise en place dans son secteur d'activité. Les auto-contrôles sont les actes fondamentaux de cette procédure mais ils ne trouvent leur place logique que dans un système global cohérent articulé sur la méthode HACCP. Cette approche globale nécessite aussi un système de veille scientifique, une définition détaillée des cahiers des charges, l'établissement de standards internes et surtout une implication forte de l'ensemble du personnel. Une telle démarche peut s'appuyer sur des services internes et/ou des aides externes telles que comités scientifiques, agences de veille, de conseil ou d'audit, instituts professionnels...

\section{Avancées et limites du système}

Un des indicateurs du niveau de sécurité des aliments en France repose sur les cas de maladie engendrée directement par les aliments. Les évolutions de ces chiffres permettent de constater que la sécurité des aliments ne cesse de se renforcer, contrairement à ce que pourrait laisser croire la transparence avec laquelle les administrations gèrent ce dossier. Les cas de listériose ont été divisés par trois entre 1987 et 1997 passant de 661 à 225, ce qui en termes d'incidence donne des valeurs très faibles : 3,8 cas par million d'habitants.

Entre 1992 et 1997, les intoxications alimentaires collectives ont oscillé entre 384 et 478 foyers par an, avec un nombre de malades variant de 7,192 à 7,858. Bien que les chiffres des TIAC soient sousestimés, il n'en demeure pas moins qu'ils ne reflètent pas une aggravation de la situation surtout si l'on tient compte de l'augmentation des tonnages de denrées alimentaires fabriquées en circulation et de la très forte augmentation des nombres de repas pris à l'extérieur. Si des progrès très importants ont été effectués dans la maîtrise des risques microbiologiques, la maîtrise des risques chimiques pose encore de nombreux problèmes. Leur prise en compte est souvent très récente du fait que la plupart des risques concernent le long terme. Ils concernent aussi l'ensemble de la chaîne alimentaire et plus souvent, la production que la transformation ou la distribution. En ce qui concerne certains produits chimiques, c'est la contamination de l'environnement qui est à l'origine de la contamination des aliments (par exemple dioxines, certains métaux lourds). La traçabilité devient alors un élément essentiel de la gestion de ces risques et doit donc pouvoir aller jusqu'à l'identification de la parcelle d'origine. De plus, l'application de la méthode HACCP aux risques chimiques se heurte à des limitations pratiques, par exemple d'ordre toxicologique pour la hiérarchisation des dangers ou d'ordre analytique pour la détectabilité. Pour certains contaminants, il n'existe pas de réglementation et, pour d'autres, l'harmonisation européenne et internationale est à peine commencée.

Un autre problème important est la gestion des risques au niveau de l'ensemble d'une population et au niveau du consommateur individuel. En effet, à l'intérieur d'une population, on peut identifier des segments de populations plus fragiles ou des segments de consommateurs extrêmes. Dans les modèles d'évaluation des risques on prend en compte le $95^{\mathrm{e}}$ percentile, c'est-à-dire que les mesures de gestion doivent assurer la sécurité de $95 \%$ de la population car on ne peut tenir compte au niveau 
national de tous les cas particuliers. Or, ces cas particuliers peuvent être relativement élevés et, de toute façon, un consommateur dont la santé est atteinte à cause de la consommation d'un aliment peut demander des comptes aux professionnels ou aux pouvoirs publics. Certains segments de la population peuvent présenter une sensibilité particulière, comme les enfants, les femmes enceintes, les personnes âgées, certains malades (immunodéprimés, insuffisants rénaux, hypersensibles aux allergènes) ou consommer des quantités très supérieures à la moyenne $(>3)$ de certains aliments. Une attention particulière doit alors être portée à ces consommateurs avec des moyens appropriés de formation, d'information (étiquetage) et de veille sanitaire. On voit donc qu'une grande partie des solutions passe par la surveillance de type analytique, biologique et épidémiologique pour sortir de débats souvent théoriques sur les dangers (par exemple effets sans seuil ou avec seuil, difficultés des études épidémiologiques pour déterminer des relations de cause à effets dans le cas d'expositions à de faibles doses de mélanges complexes).

Notes:

${ }^{1} 10^{-15} \mathrm{~g}$.

${ }^{2} 1 \mathrm{pg}=10^{-12} \mathrm{~g}$.

\section{CONCLUSION}

On peut donc conclure que la sécurité des aliments est fondée sur trois piliers fondamentaux : l'évaluation des risques, la traçabilité du champ au consommateur et la surveillance. Nous sommes en train de vivre une véritable révolution dans l'évaluation et la gestion des risques, qui change fondamentalement les liens entre les citoyens, les opérateurs, les pouvoirs publics sans oublier les médias (éléments essentiels de la communication) et les structures d'éducation, le corps médical et la recherche, en mettant le consommateur au centre du système.

\section{REFERENCES}

\{references\} 